\title{
Gilbert Syndrome
}

National Cancer Institute

\section{Source}

National Cancer Institute. Gilbert Syndrome. NCI Thesaurus. Code C84729.

An autosomal recessive inherited disorder characterized by unconjug ated

hyperbilirubinemia, resulting in harmless intermittent jaundice. 